\title{
REDUCING LATENCY WHEN USING VIRTUAL REALITY FOR TEACHING IN SPORT
}

\author{
Yulita Hanum P Iskandar, Lester Gilbert, Gary B Wills \\ Learning Technologies Research Group \\ Electronics \& Computer Science, University of Southampton, SO17 IBJ, United Kingdom. \\ $\{y$ hi07r, lg3, gbw\}@ecs.soton.ac.uk
}

\begin{abstract}
Latency is a frequently cited shortcoming of Virtual Reality (VR) applications. To compensate for excessive latency, prediction mechanisms may use sophisticated mathematical algorithms, which may not be appropriate for complex virtual teaching applications. This paper suggests that heuristic prediction algorithms could be used to develop more effective and general systems for VR educational applications. A fast synchronization squash simulation illustrates where heuristic prediction can be used to deal with latency problems.
\end{abstract}

\section{Introduction}

In recent years, the field of Virtual Reality (VR) has grown significantly. The development of VR is a prevalent and growing phenomenon in domains such as scientific visualization, medical diagnosis and surgical training, flight simulation, educational tools, and 3D games. Proponents of educational VR believe that this is the future of instruction for training and teaching in sports.

Teaching a sport virtually needs more than just the story, the graphic artwork, and the software. It needs appropriate pedagogy, where the activities support the instructional strategy. Well executed virtual sport teaching needs to combine the cognitive and sensorymotor domains. Coaches are trying to give better feedback to athletes by using computer technology to improve performance.

In Things That Make Us Smart, Donald Norman identifies seven basic requirements of a learning environment (Norman, 1993, cited in [1]). Relevant to this paper is the requirement of providing a high intensity of interaction and feedback.

Current theories of motor learning support the notion that skills are developed through practice and feedback mechanisms that allow a generalized motor program to be developed for related skills. Feedback is necessary regardless of the stage of development of the athlete. According to [2], in order for the feedback to be most effective and usable, it needs to be immediate, specific and contingent.

However, the requirement of such feedback is not easily met. One of the critical problems is the perceived latency or lag [3-5]. Human users of virtual environments (VEs) are disturbed by system latency, which limits their usefulness in real world applications $[6,7]$. Latency, in this context, refers to the delay that occurs between the movement of the user within a VE and the result of that action being reflected by the VE.

This paper is organized into five sections: 1) discussion of the latency problem as one of delayed feedback, 2) review of some previous work in handling latency problems, 3) introduction of an example of a sport simulation, 4) proposed solution for latency problems, and finally 5) reflection and conclusions from this research.

\section{Problem in delaying feedback}

In virtual teaching applications, one of the major issues is to provide an immersive environment, which is computer generated with realistic appearance, behavior, and interaction. The computer needs time to read the tracker measurements, set the new camera position, perform rendering, etc. Because of this, pictures are presented with some delay, which makes some "especially fast" tasks harder to perform.

The total latency of a virtual sport system may be defined as the delay between the movement of the real objects (e.g. the racket) and the corresponding visible feedback at the screen. There are two fundamental issues that affect speed and latency requirements. The first concerns the sensor values, which are the most sensitive to performance. The second concerns the individual components that combine to produce total system lag. 


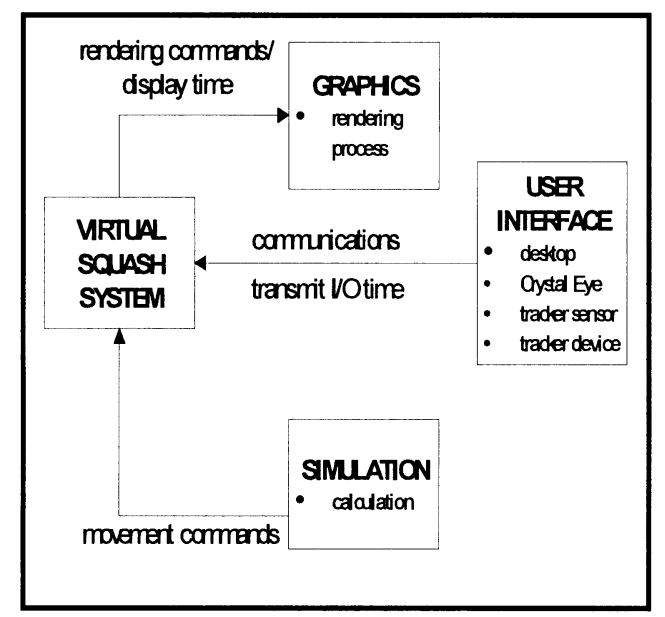

Figure 1. Illustration of latency in virtual sport system

Figure 1 illustrates the system components that contribute to the time delay between the time sensors are read and the time an appropriate scene is rendered. The total latency of this system is the sum of the latencies of the sub components of the system (strictly, that lie on the critical path). Overall system latency and its visible consequences are fundamental $\mathrm{VE}$ deficiencies that can hamper user perception and performance. For example, latency in estimating head orientation of only a few milliseconds can at best be distracting and at worst lead to cyber-sickness. At the same time, the illusion of a virtual world is destroyed if the objects on the screen jitter significantly while the head is not in motion (the "swimming effect"). Similarly, latency in other parts of a virtual human body can make the system difficult to interact with and used effectively.

Excessive latency has been known to degrade manual performance, forcing users to slow down to preserve manipulative stability and ultimately driving them to adopt "move and wait" strategies. This problem has proven to be the most limiting when trying to use VR as an educational tool for real-world applications. For example, in a virtual teaching environment, when a trainee moves to an actual system with different latency characteristics, some of the techniques and strategies developed in the VE may not successfully transfer, making the VR education less successful.

Moreover, the effectiveness of teaching and learning in VE is dependent on the realism of the system [5, 8]. The realism of a VE is improved by three-dimensional design, high fidelity surround sound, and high resolution graphics at smooth rendering rates. Realism links back to internal feedback experienced by the trainee. Such internal feedback is a major contribution to motivation and to trainees' evaluation of their movements. In this situation, trainees will most effectively feel, see, and hear the consequences of their actions. According to [8], only when users "believe" or "immerse" in their environment, forgetting that all surrounding objects are virtual, do they take the environment so seriously that they engage in full body activities and may sweat as they do in a real sport.

\section{Previous work with handling latency}

Research into reducing the detrimental effects of latency can be categorized as bounding latency, reducing latency, compensating for latency, and achieving registration despite latency [9]. However, none of this has been entirely successful.

Reducing latency depends on improving the hardware tracking sensors. Latency can be also be reduced by improving the software generating the virtual world. Most current approaches for VR focuses on polygon rendering, which involves approximating the shape of each object with various numbers of polygons. Polygon rendering can only give real-time performance for simple virtual worlds; to handle richer worlds, improved algorithms must come from somewhere else. "Time-critical" computation, essential for VR systems to give real-time performance [10], is a technique in which quality is traded for speed. Latency is reduced by increasing the speed of the required computations $[4,5]$. To reduce the latency through system hardware and software reorganization, [11] improved temporal response significantly by minimizing round trip delays.

Hardware improvements alone, however, cannot solve the problem. This is because computation and sensor and display processing take finite time to execute and involve minimum latency no matter how small. The best results up to now have been achieved by the prediction method [12]. Prediction based systems use signals from 3D trackers as the inputs, process them, and output the predicted data. Processing takes place in the interaction cycle between tracking and evaluation stages. Very often in any application, motion position has to be predicted well in advance in order to overcome delays that are introduced by the image generating system. Without prediction, VR must use the current pose to compute and display new images for each frame. This simple approach can cause problems since the user might be moving during the computation and display processes, resulting in stale images and a display-to-user-motion synchronization mismatch. 
Table 1. Categorization of prediction algorithms

\begin{tabular}{|l|l|}
\hline \multicolumn{2}{|c|}{ PREDICTION ALGORITHM } \\
\hline Interpolation & Lagrangian Polynomials \\
\hline \multirow{4}{*}{ Extrapolation } & Lagrangian Polynomials \\
\cline { 2 - 2 } & Orthogonal Polynomials \\
\cline { 2 - 2 } & Eulerien Lagrangian \\
\cline { 2 - 2 } & Cubic Splines \\
\cline { 2 - 2 } Integerized & $2^{\text {nd }}$ Order Taylor Expansions \\
\hline \multirow{5}{*}{ Filter-based } & Grey System \\
\hline \multirow{5}{*}{ Neural Network } & Kalman Filter \\
\cline { 2 - 2 } & Extended Kalman Filter \\
\cline { 2 - 2 } & Unscented Kalman Filter \\
\cline { 2 - 2 } & Particle Filer \\
\cline { 2 - 2 } & Wiener Filter \\
\cline { 2 - 2 } & Grid-Based Filter \\
\cline { 2 - 2 } & Linear-Time-Invariant \\
\cline { 2 - 2 } & Multiple Model Adaptive \\
& Estimation \\
\cline { 2 - 2 } & Perceptron Neural Network \\
\cline { 2 - 2 } & Back-Propagation Network \\
\cline { 2 - 2 } & Elman Neural Network \\
\hline
\end{tabular}

Table 1 shows the different categories of prediction algorithms which have demonstrated the ability to track human movement in VR environments. However, each of the prediction algorithms has its own limitations. Any prediction algorithm involves a complex mathematical algorithm, such that more complex VR simulations may impose greater computational burdens and result in the increase of latency [13]. Furthermore, according to [12], almost all prediction algorithms contain one or more parameters that are used for tuning to optimize performance and a significant aspect in selecting an appropriate prediction algorithms depends on successfully adjusting an algorithm's parameter values. These adjustments are nontrivial in the sense that an optimal parameter setting for one type of user motion may not be optimal for another.

Introducing heuristics is potentially one way forward in dealing with the latency problem. Human motion sequences may be considered to be comprised "primitive motions" [14]. A primitive motion consists of a basic motion which is common to all players. Human motion cannot be modeled exactly by mathematical or physical methods [15]. And, as sport is a game of habits, during sport a player typically acts in a certain habitual pattern.

In training a free motion, the kinesthetic aspect of an action is important [16]. In developing a robust prediction algorithm, it is important to achieve good estimates of the motion kinematics. Humans have developed a set of powerful heuristics for planning motion by solving motion planning problems whenever they move [17]. For this purpose, we may consider the implementation of heuristically predicted motion.

\section{Theoretical analysis of a squash simulation}

Sports training applications are good potential examples of the dynamic movements required in VR training. In order to find out whether the proposed heuristic prediction algorithms are acceptable and could fulfill the main purpose of reducing latency in VR educational applications; this paper has chosen squash as a study source. According to [18]: "One rally may last one stroke and 1.5 seconds, or 400 strokes and 10 minutes. More usually - for training purposes rallies can be categorized into three populations, those lasting less than 5 seconds, those between 6-20 seconds, and a small but important number which last upwards of 20, but not usually longer than 60 seconds. There are approximately 7 seconds between rallies, which are played at a rate of up to 40 strokes per minute."

In general, virtual sports environments are very difficult to construct in comparison with other virtual tasks because the motion speed of players is very fast and the motion area is quite large. To realize a squash simulation, high speed motion prediction is required. Furthermore, a high-speed visual system with stereovision is necessary so as to display a moving player with high speed.

Speed and latency are probably the two most important characteristics affecting a comfortable and believable VR educational tool. Based on the analysis of latency components and the squash game itself which involves very fast movements, a squash simulation needs effective prediction algorithms.

\section{Proposed Solution}

This paper proposes a prediction method based on heuristics that could be used to develop a more effective and general system for VR educational applications. According to Perkins (1981 cited in [19]), a heuristic is: "A rule of thumb that often helps in solving a certain class of problems but makes no guarantees."

Although heuristic algorithms have been applied to a wide range of humanoid robots and avatars in VR systems [20,21], there seem to be no published reports that the suggested approach has been tried as a single prediction algorithm for compensating latency in VR educational applications. 
A heuristic approach introduces a motion control paradigm that simplifies the generation of expressive movements by proceduralizing the qualitative aspects of movement. There are five fundamental principles on movement [22]:

1. Movement is a process of change.

2. The change is patterned and orderly.

3. Human movement is intentional.

4. The basic elements of human movement may be articulated and studied.

5. Movement must be approached at multiple levels if it is to be properly understood.

These movement principles can be considered a heuristic. This is because human movement can be patterned and orderly which is also known as a "rule of thumb". The heuristic prediction algorithm for a squash VR system would thus focus on the habitual motion of the players in a squash game, which has certain rules, tactics, and patterns of movement that makes it an ideal subject for this.

It has been suggested that individuals may use heuristics of reasoning and rely on schemata when a quick decision is necessary. Decisions made by a squash player are very likely to be influenced by prior knowledge. Squash is a type of game with fast reactions as well as coordination, concentration, and anticipation [23-25]. We would expect heuristics to be involved in a squash VR application concerned with the automation of intelligent behavior.

Many heuristic planners have been created in an attempt to achieve the necessary speeds in off-line (or more ambitiously, on-line) processing [26], and these would be expected to have useful application to the design of VR programs that would simulate human behavior. For many applications, a specialized heuristic motion planner is often sufficient and a computationally expensive complete planner may not be needed.

A heuristic prediction algorithm for kinematics would combine natural and unobtrusive interaction through gesturing with precise and general-purpose interaction. The basic heuristic-based algorithm is to move the player from a start location to a goal location, thus minimizing the path length in terms of time.

Motion planning incorporates path prediction with rules on human motion limitations (e.g. angular reach). Effective path prediction requires an analysis of the habitual human behavior in the specific domain of the sport application (e.g. motion of squash players). Motion planning can then update the player's motion in a way which can be referred to as heuristic-based prediction.

\section{Conclusion}

The potential for heuristic-based prediction algorithms in VR, educational, and sport applications have been derived from a theoretical analysis of latency, feedback, existing algorithms, and consideration of a specific sport, squash.

The paper argues for the benefits of heuristic over previous prediction algorithms and what is now needed is empirical evidence. On-going research is being planned for experiments to validate heuristic predictions using data from a variety of tracking systems, applications and interaction techniques. We believe that the results may extend to other domains in teaching and training applications, to robotics, and to applications involving animation.

\section{References}

[1]. Houser, R. and S. DeLoach. Instructional design lessons technical communicators can learn from games. in Professional Communication Conference, 1996. IPCC '96 Proceedings. 'Communication on the Fast Track', International. 1996.

[2]. Gilbert, L. and V. Gale, Principles of E-learning Systems Engineering. 2008: Chandos.

[3]. Adelstein, B.D., T.G. Lee, and S.R. Ellis. Head Tracking Latency in Virtual Environments: Psychophysics and a model. in Proceedings of the Human Factors and Ergonomics Society 47th Annual Meeting. 2003.

[4]. Wu, J.-R. and M. Ouhyoung, On latency compensation and its effects on head-motion trajectories in virtual environments The Visual Computer, 2004. 16: p. 79-90

[5]. Kyger, D.W. and P.S. Maybeck, Reducing lag in virtual displays using multiple model adaptive estimation. Aerospace and Electronic Systems, IEEE Transactions on, 1998. 34(4): p. 1237-1248.

[6]. LaViola, J.J., Jr. A comparison of unscented and extended Kalman filtering for estimating quaternion motion. in American Control Conference, 2003. Proceedings of the 2003. 2003.

[7]. Van Rhijn, A., R. Van Liere, and J.D. Mulder. $A n$ analysis of orientation prediction and filtering methods for VR/AR. in Virtual Reality, 2005. Proceedings. VR 2005. IEEE. 2005.

[8]. Sari, M., et al., Fitness computer game with a bodily user interface, in Proceedings of the second international conference on Entertainment computing. 2003, Carnegie Mellon University: Pittsburgh, Pennsylvania.

[9]. Kiruluta, A., M. Eizenman, and S. Pasupathy, Predictive head movement tracking using a Kalman filter. Systems, Man, and Cybernetics, Part B, IEEE Transactions on, 1997. 27(2): p. 326-331. 
[10]. Jiann-Rong, W. and O. Ming, A 3D tracking experiment on latency and its compensation methods in virtual environments, in Proceedings of the 8th annual ACM symposium on User interface and software technology. 1995, ACM: Pittsburgh, Pennsylvania, United States.

[11]. Jacoby, R.H., B.D. Adelstein, and S.R. Ellis. Improved Temporal Response in Virtual Environments through System Hardware and Software Reorganization. in Proceedings of SPIE: Stereoscopic Displays and Virtual Reality Systems III. 1996.

[12]. Joseph, J.L., A testbed for studying and choosing predictive tracking algorithms in virtual environments, in Proceedings of the workshop on Virtual environments 2003. 2003, ACM: Zurich, Switzerland.

[13]. Jung, J.Y., B.D. Adelstein, and S.R. Ellis. Discriminability of Prediction Artifacts in a TimeDelayed Virtual Environment. in Human Factors and Ergonomics Society Annual Meeting Proceedings. 2000: Human Factors and Ergonomics Society.

[14]. Nakazawa, A., et al. Analysis and synthesis of human dance motions. in Multisensor Fusion and Integration for Intelligent Systems, MFI2003. Proceedings of IEEE International Conference on. 2003.

[15]. Shihong, X., Q. Xianjie, and W. Zhaoqi, A novel framework for athlete training based on interactive motion editing and silhouette analysis, in Proceedings of the ACM symposium on Virtual reality software and technology. 2005, ACM: Monterey, CA, USA.

[16]. Baek, S., S. Lee, and G.J. Kim, Motion retargeting and evaluation for VR-based training of free motions. The Visual Computer, 2003. 19(4): p. 222-242.

[17]. Hwang, Y.K., et al. Human computer cooperation in interactive motion planning. in Advanced Robotics, 1997. ICAR '97. Proceedings., 8th International Conference on. 1997.

[18]. Sharp, C. Fitness Formula 1993 [cited 200820 January]; Available from: http://www.squashplayer.co.uk/fitness factory. htm.

[19]. Plsek, P.E. The Basic Heuristics of DirectedCreativity. 1997 [cited 20071 December 2007]; Available from: http://www.directedereativity.com/pages/Heuristics html\#PerkinsRef.

[20]. Tsuji, T., Y. Takeda, and Y. Tanaka. Analysis of mechanical impedance in human arm movements using a virtual tennis system in Biological Cybernetics. 2004: Springer.

[21]. Victor Brian, Z. and K.H. Jessica, Motion capturedriven simulations that hit and react, in Proceedings of the 2002 ACM SIGGRAPH/Eurographics symposium on
Computer animation. 2002, ACM: San Antonio, Texas.

[22]. Price, H., Time's Arrow and Archimedes' Point: New Directions for the Physics of Time. 1996, Oxford University Press.

[23]. Colin, W. and B. Ravin, Reaching for objects in VR displays: lag and frame rate. 1994, ACM. p. 331356.

[24]. MacKenzie, I.S. and W. Colin, Lag as a determinant of human performance in interactive systems, in Proceedings of the INTERACT '93 and CHI '93 conference on Human factors in computing systems. 1993, ACM: Amsterdam, The Netherlands.

[25]. Vuckovic, G., et al. Motion analysis of the international and national rank squash players. in Image and Signal Processing and Analysis, 2005. ISPA 2005. Proceedings of the 4th International Symposium on. 2005.

[26]. Wan, T.R., H. Chen, and R. Earnshaw. Real-time path planning for navigation in unknown environment. in Theory and Practice of Computer Graphics, 2003. Proceedings. 2003. 